following lower bounds for $\bar{x}$ and $\phi(\bar{x})$ : (I) $10^{458}$; (II) $10^{586}$; (III) $10^{400}$.

\title{
REFERENCES
}

1. R. D. Carmichael, Note on Euler's $\phi$-function, Bull. Amer. Math. Soc. vol. 28 (1922) pp. 109-110.

2. D. N. Lehmer, List of prime numbers, Carnegie Institution Publication, no. 165.

UNIVERSITY OF VIRGINIA

\section{ON THE DARBOUX TANGENTS}

V. G. GROVE

1. Introduction. In a recent paper [1] ${ }^{1}$ Abramescu gave a metr1cal characterization of the cubic curve obtained by equating to zero the terms of the expansion of a surface $S$ at an ordinary point $O_{1}$, up to and including the terms of the third order. This cubic curve is rational and its inflexions lie on the three tangents of Darboux through $O_{1}$. In this paper we give a projective characterization of such a curve, and hence a new derivation of the tangents of Darboux. By using the method employed in this characterization to the curve of intersection of the tangent plane of the surface at $O_{1}$ with $S$, a simple characterization of the second edge of Green is found. Another application exhibits the correspondence of Moutard. Finally a new interpretation of the reciprocal of the projective normal is given in terms of the conditions of apolarity of a cubic form to a quartic form. The canonical tangent appears in a similar fashion.

Let $S$ be referred to its asymptotic curves, and let the coordinates $\left(x^{1}, x^{2}, x^{3}, x^{4}\right)$ of the generic point $O_{1}$ of $S$ be normalized so that they satisfy the system [2] of differential equations

$$
\begin{aligned}
x_{u u} & =\theta_{u} x_{u}+\beta x_{v}+p x, \\
x_{v v} & =\gamma x_{u}+\theta_{v} x_{v}+q x, \quad \theta=\log R .
\end{aligned}
$$

The line $l_{1}$ joining $O_{1}$ to $O_{4}$, whose coordinates are $x_{u v}^{i}$, is the $R$-conjugate line, and the line $l_{2}$ determined by $O_{2}, O_{3}$, whose respective coordinates are $x_{u}^{i}, x_{v}^{i}$, is the $R$-harmonic line.

If we define the local coordinates $\left(x_{1}, x_{2}, x_{3}, x_{4}\right)$ with respect to

Presented to the Society, April 26, 1947; received by the editors April 11, 1947.

${ }^{1}$ Numbers in brackets refer to the references cited at the end of the paper. 
$\mathrm{O}_{1} \mathrm{O}_{2} \mathrm{O}_{3} \mathrm{O}_{4}$ of a point $X$ by the expression

$$
X^{i}=x_{1} x^{i}+x_{2} x_{u}^{i}+x_{3} x_{v}^{i}+x_{4} x_{u v}^{i},
$$

and local nonhomogeneous coordinates $(x, y, z)$ by $x=x_{2} / x_{1}, y=x_{3} / x_{1}$, $z=x_{4} / x_{1}$, the power series expansion [4] of $S$ at $O_{1}$ is

$$
z=x y-\frac{1}{3}\left(\beta x^{3}+\gamma y^{3}\right)+\frac{1}{12} F_{4}(x, y)+\cdots,
$$

wherein

$$
\begin{aligned}
F_{4}(x, y)= & \left(2 \beta \theta_{u}-\beta_{u}\right) x^{4}-4\left(\beta \theta_{v}+\beta_{v}\right) x^{3} y-6 \theta_{u v} x^{2} y^{2} \\
& -4\left(\gamma \theta_{u}+\gamma_{u}\right) x y^{2}+\left(2 \gamma \theta_{v}-\gamma_{v}\right) y^{4} .
\end{aligned}
$$

2. Characteristic points of a plane curve. Let the triangle of reference $O_{1} O_{2} O_{3}$ to which a plane curve $C$ is referred be covariant to the curve or to a surface to which $C$ bears some geometrical relation. Let the homogeneous coordinates of a point with respect to this triangle be $\left(x_{1}, x_{2}, x_{3}\right)$, the nonhomogeneous coordinates being defined by the expressions $x=x_{2} / x_{1}, y=x_{3} / x_{1}$. The line $y=0$ being chosen as the tangent to $C$ at $O_{1}$, the power series expansion [4] of $C$ at $O_{1}$ is

$$
y=a_{2} x^{2}+a_{3} x^{3}+a_{4} x^{4} \cdots .
$$

Consider at $O_{3}(0,0,1)$ the involution whose double lines are $O_{1} O_{3}$, $\mathrm{O}_{2} \mathrm{O}_{3}$. Corresponding lines of this involution intersect $C$ in points $P_{1}(x, y), P_{2}\left(-x, y^{\prime}\right), y^{\prime}=a_{2} x^{2}-a_{3} x^{3}+a_{4} x^{4}-\cdots$. The line $P_{1} P_{2}$ intersects the tangent to $C$ at $O_{1}$ in a point whose limit $T$ as $P_{1}$ approaches $O_{1}$ along $C$ has coordinates

$$
x_{1}=a_{3}, \quad x_{2}=-a_{2}, \quad x_{3}=0 .
$$

We shall call the point $T$ with coordinates (2.2) the characteristic point of the second order of $\mathrm{C}$ at $\mathrm{O}_{1}$ relative to $\mathrm{O}_{1} \mathrm{O}_{3} \mathrm{O}_{2}$.

Let $O_{2}^{\prime}(\rho, 1,0)$ be an arbitrary point on the tangent to $C$ at $O_{1}$, but distinct from $O_{1}$. The transformation from the triangle $O_{1} O_{2} O_{3}$ to $O_{1} O_{2}^{\prime} O_{3}$ is

$$
x=\frac{A x^{\prime}}{1+\rho A x^{\prime}}, \quad y=\frac{B y^{\prime}}{1+\rho A x^{\prime}} .
$$

Under the transformation (2.3), the equation of $C$ may be written in the form

$$
y^{\prime}=a_{2}^{\prime} x^{\prime 2}+a_{3}^{\prime} x^{\prime 3}+\cdots,
$$

wherein 


$$
a_{2}^{\prime}=A^{2} a_{2} / B, \quad a_{3}^{\prime}=A^{3}\left(a_{3}-\rho a_{2}\right) / B .
$$

Hence the characteristic point of $\mathrm{C}$ relative to $\mathrm{O}_{1} \mathrm{O}_{3} \mathrm{O}_{2}^{\prime}$ has coordinates

$$
x_{1}=\left(a_{3}-2 \rho a_{2}\right), \quad x_{2}=-a_{2}, \quad x_{3}=0
$$

referred to $\mathrm{O}_{1} \mathrm{O}_{2} \mathrm{O}_{3}$.

More generally let the equation of $C$ have the form

$$
y=a_{k} x^{k}+a_{k+1} x^{k+1}+\cdots, \quad k \geqq 2 .
$$

Consider through $\mathrm{O}_{3}$ two lines forming with $\mathrm{O}_{1} \mathrm{O}_{3}, \mathrm{O}_{2} \mathrm{O}_{3}$ the constant cross ratio $l, l$ being one of the $k$ th roots of unity, but $l \neq 1$. These lines intersect $C$ in two points $P_{1}, P_{2}$ determining a line which intersects the tangent to $C$ at $O_{1}$ in a point whose limit as $P_{1}$ approaches $O_{1}$ has coordinates

$$
x_{1}=a_{k+1}, \quad x_{2}=-a_{k}, \quad x_{3}=0 .
$$

We shall call the point $T$ whose coordinates are (2.5) the characteristic point of the kth order of $\mathrm{C}$ relative to $\mathrm{O}_{1} \mathrm{O}_{3} \mathrm{O}_{2}$.

3. The characteristic curve of $S$. Let us consider the section $C_{x}$ of the surface $S$ by a plane $\pi$ through the $R$-conjugate line $l_{1}$. Let $\pi$ intersect the $R$-harmonic line $l_{2}$ in $O_{\pi}$. The local coordinates of $O_{\pi}$ are of the form $(0, \lambda, \mu, 0)$, and the local coordinates of any point $Q_{1}$ on $O_{1} O_{\pi}$ are $(1, \lambda \xi, \mu \xi, 0)$. The equation of $C_{\pi}$ referred to $O_{1} O_{\pi} O_{4}$ in nonhomogeneous coordinates $(\xi, z)$ is

$$
z=\lambda \mu \xi^{2}-\frac{1}{3}\left(\beta \lambda^{3}+\gamma \mu^{3}\right) \xi^{3}+\frac{1}{12} F_{4}(\lambda, \mu) \xi^{4}+\cdots
$$

From (2.2) the characteristic point $T_{\pi}$ of $C_{\pi}$ relative to $O_{1} O_{4} O_{\pi}$ has coordinates

$$
\xi=3 \lambda \mu /\left(\beta \lambda^{3}+\gamma \mu^{3}\right), \quad z=0,
$$

referred to $\mathrm{O}_{1} \mathrm{O}_{\pi} \mathrm{O}_{4}$, and coordinates

$$
x=3 \lambda^{2} \mu /\left(\beta \lambda^{3}+\gamma \mu^{3}\right), \quad y=3 \lambda \mu^{2}\left(\beta \lambda^{3}+\gamma \mu^{3}\right), \quad z=0
$$

referred to $\mathrm{O}_{1} \mathrm{O}_{2} \mathrm{O}_{3} \mathrm{O}_{4}$. The locus of $T_{\pi}$ as $\pi$ rotates about $l_{1}$ is the covariant rational cubic curve $\Gamma_{3}$ whose equation is

$$
3 x y-\left(\beta x^{3}+\gamma y^{3}\right)=0, \quad z=0 .
$$

We shall call this cubic the characteristic curve of $S$ relative to $l_{1}, l_{2}$. The nodal tangents of $\Gamma_{3}$ are of course the asymptotic tangents of $S$ at $O_{1}$, and the inflexions lie on the tangents of Darboux. The $R$-harmonic line 
is the flex-ray of $\Gamma_{3}$.

From (3.3) it follows that the only sections of $S$ through the $R$-conjugate line whose characteristic points relative to $\mathrm{O}_{1} \mathrm{O}_{4} \mathrm{O}_{\pi}$ lie on the $R$-harmonic line are those through the tangents of Darboux.

Another characterization of the cubic $\Gamma_{3}$ may be found in the following manner. The osculating conic of the section $C_{\pi}$ has the equation [4]

$$
\begin{aligned}
\lambda^{3} \mu^{3}\left(z-\lambda \mu \xi^{2}\right)+\frac{1}{3} & \lambda^{2} \mu^{2}\left(\beta \lambda^{3}+\gamma \mu^{3}\right) \xi z \\
+ & {\left[\frac{1}{9}\left(\beta \lambda^{3}+\gamma \mu^{3}\right)^{2}-\frac{1}{12} F_{4}(\lambda, \mu)\right] z^{2}=0 }
\end{aligned}
$$

The pole of $R$-conjugate line with respect to this conic is the point $T_{\pi}^{\prime}$ with coordinates

$$
\xi=-3 \lambda \mu /\left(\beta \lambda^{3}+\gamma \mu^{3}\right), \quad z=0 .
$$

The harmonic conjugate of $T_{\pi}^{\prime}$ with respect to $O_{1} O_{\pi}$ is the point $T_{\pi}$ defined by (3.2). Incidentally the locus of $T_{\pi}^{\prime}$ is the cubic $\Gamma_{3}^{\prime}$,

$$
3 x y+\beta x^{3}+\gamma y^{3}=0 .
$$

The tangents of Darboux are thus again exhibited by means of $\Gamma_{3}^{\prime}$.

Finally we may readily show that the polar line of the conic (3.5) intersects $\mathrm{O}_{4} \mathrm{O}_{\boldsymbol{x}}$ in a point whose locus as $\pi$ varies is a rational curve of order seven which intersects the $R$-harmonic line at its intersections with the tangents of Darboux.

4. The edges of Green. The expansions [4] of the two branches of the curve of intersection of $S$ at $O_{1}$ with its tangent plane are

$$
\begin{aligned}
& y=\frac{1}{3} \beta x^{2}-\frac{1}{12}\left(2 \beta \theta_{u}-\beta_{u}\right)^{3}+\cdots, z=0 ; \\
& x=\frac{1}{3} \gamma y^{2}-\frac{1}{12}\left(2 \gamma \theta_{v}-\gamma_{v}\right)^{3}+\cdots, z=0 .
\end{aligned}
$$

The characteristic point $T_{u}$ of the first of (4.1) relative to $O_{1} O_{3} O_{2}$ has coordinates

$$
x_{1}=\frac{1}{4}\left(2 \theta_{u}-\frac{\beta_{u}}{\beta}\right), \quad x_{2}=1, \quad x_{3}=x_{4}=0,
$$

and the characteristic point $T_{v}$ of the second relative to $\mathrm{O}_{1} \mathrm{O}_{2} \mathrm{O}_{3}$ has coordinates 


$$
x_{1}=\frac{1}{4}\left(2 \theta_{v}-\frac{\gamma_{v}}{\gamma}\right), \quad x_{2}=0, \quad x_{3}=1, \quad x_{4}=0 .
$$

The line joining the harmonic conjugates of $T_{u}$ and $T_{v}$ with respect to $\mathrm{O}_{1} \mathrm{O}_{2}$ and $\mathrm{O}_{1} \mathrm{O}_{3}$ respectively is Green's edge of the second kind.

This edge of Green may be characterized in another way. The section of $S$ by the plane through the $R$-conjugate line and the tangent to the asymptotic curve $v=$ const. has the equation

$$
z=-\frac{1}{3} \beta x^{3}+\frac{1}{12}\left(2 \beta \theta_{u}-\beta_{u}\right) x^{4}+\cdots .
$$

The characteristic point of the third order of the curve (4.4) relative to $\mathrm{O}_{1} \mathrm{O}_{4} \mathrm{O}_{2}$, is found from (2.5) to have coordinates given by (4.2); by interchanging the roles of the asymptotic tangents the point (4.3) is characterized. The second edge of Green is therefore given another characterization.

Consider on the tangent to the section (3.1) $C_{\pi}$ of $S$ the point $O_{\pi}^{\prime}(\rho, 2 \lambda, 2 \mu, 0)$. From $(2.4)$ we find readily that the characteristic point $T$ of $C_{\pi}$ relative to $\mathrm{O}_{1} \mathrm{O}_{4} \mathrm{O}_{\pi}^{\prime}$ has coordinates

$$
x_{1}=\rho \lambda \mu+\frac{1}{3}\left(\beta \lambda^{3}+\gamma \mu^{3}\right), \quad x_{2}=\lambda^{2} \mu, \quad x_{3}=\lambda \mu^{2}, \quad x_{4}=0 .
$$

The point $P_{\pi}$ on the tangent to $C_{\pi}$ at $O_{1}$ which with $O_{1}$ separates $O_{\pi}^{\prime}$ and $O_{\pi}$ harmonically has coordinates $(\rho, \lambda, \mu, 0)$. Equations (4.4) therefore represent a cubic transformation of $P_{\pi}$ into the characteristic point of $C_{\pi}$ relative to $\mathrm{O}_{1} \mathrm{O}_{4} \mathrm{O}_{\pi}^{\prime}$. The polar plane of the point (4.5) with respect to any quadric of Darboux,

$$
x_{2} x_{3}-x_{1} x_{4}+k_{4} x_{4}^{2}=0
$$

has coordinates

(4.6) $\quad \xi_{1}=0, \quad \xi_{2}=\lambda \mu^{2}, \quad \xi_{3}=\lambda^{2} \mu, \quad \xi_{4}=-\rho \lambda \mu-\frac{1}{3}\left(\beta \lambda^{3}+\gamma \mu^{3}\right)$.

The correspondence (4.6) between $P_{\pi}$ and the polar plane of the characteristic point of $C_{\pi}$ relative to $\mathrm{O}_{1} \mathrm{O}_{4} \mathrm{O}_{\pi}{ }^{\prime}$ is the correspondence of Moutard $(k=-1 / 3)$. We have previously [3] given a different derivation of this correspondence.

5. The projective normal. The surface $S^{\prime}$ whose equation is

$$
z=x y-\frac{1}{3}\left(\beta x^{3}+\gamma y^{3}\right)
$$


has a unode at $O_{4}$, the plane $O_{2} O_{3} O_{4}$ as uniplane, and has contact of the third order with $S$ at $O_{1}$; hence $S^{\prime}$ is completely determined. The projection on their common tangent plane at $O_{1}$ of the curve of intersection of $S$ and $S^{\prime}$ has a quadruple point at $O_{1}$, the quadruple tangents being given by

$$
F_{4}(x, y)=0
$$

where $F_{4}(x, y)$ is defined by (1.3). The lines (5.2) intersect the $R$-harmonic line in four points $F_{i}$, and the Segre tangents intersect this line in three points $S_{i}$. It is easy to verify that the points $S_{i}$ are apolar to $F_{i}$ if and only if the $R$-harmonic line is the reciprocal of the projective normal. The projective normal is therefore geometrically determined by reciprocation with respect to the quadrics of Darboux.

Finally let the lines $l_{1}, l_{2}$ be the projective normal and its reciprocal; then it readily follows that the polar of the form $\beta x^{3}+\gamma y^{3}$ with respect to $F_{4}(x, y)$ is

$$
\phi x-\psi y
$$

wherein $\phi=\partial \log \left(\beta \gamma^{2}\right) / \partial u, \psi=\partial \log \left(\beta^{2} \gamma\right) / \partial v$. The form (5.3) equated to zero is seen to be the equation of the canonical tangent.

\section{REFERENCES}

1. N. Abramescu, Sur les tangentes de Darboux d'une surface, Annales Scientifiques Universitatea Jassey, Section I vol. 27 (1941) pp. 283-288.

2. V. G. Grove, On canonical forms of differential equations, Bull. Amer. Math. Soc. vol. 36 (1930) pp. 582-586.

3. - The transformation of Čech, Bull. Amer. Math. Soc. vol. 50 (1944) pp. 231-234.

4. E. P. Lane, A treatise on projective differential geometry, The University of Chicago Press, 1942.

Michigan State College 